\title{
EBV-positive post-transplant lymphoproliferative disorder presenting as primary diffuse large B-cell lymphoma of the central nervous system
}

\author{
Haoliang $\mathrm{Xu}^{1}$, Julia Rewerska ${ }^{1}$, Nathan Aardsma ${ }^{1}$, Konstantin Slavin ${ }^{2}$, Tibor Valyi-Nagy ${ }^{1}$, Hongyu $\mathrm{Ni}^{1}$ \\ ${ }^{1}$ Department of Pathology, University of Illinois at Chicago, Chicago, ${ }^{2}$ Department of Neurosurgery, University of Illinois at Chicago, \\ Chicago, USA
}

Folia Neuropathol 2017; 55 (3): 221-226

DOI: https://doi.org/10.5114/fn.2017.70487

\begin{abstract}
Primary central nervous system post-transplant lymphoproliferative disorder (PCNS-PTLD) is a rare complication with inferior survival outcomes in solid organ transplant patients. It represents approximately 7-15\% of all PTLD patients. Because of the rarity of this disease, the diagnosis of PCNS-PTLD is often challenging, and the optimal therapy has not been established. We report a case of a renal transplant patient who initially presented with acute altered neurological function, an enhancing mass lesion of the brain on magnetic resonance imaging (MRI), and nonspecific reactive histopathological changes on brain biopsy. The lesion was self-limited and spontaneously resolved without medical treatment for PTLD. Six months later, surveillance MRI revealed recurrence of the brain lesion. The biopsy showed morphologic changes consistent with Epstein-Barr virus (EBV)-positive diffuse large B-cell lymphoma. The patient responded well to reduction of immunosuppression and treatment with a single-agent regimen of rituximab. This is an unusual case of PCNS-PTLD with an initial presentation resembling a self-limited reactive lesion.
\end{abstract}

Key words: post-transplant lymphoproliferative disorder, Epstein-Barr virus, large B-cell lymphoma, central nervous system.

\section{Introduction}

Post-transplant lymphoproliferative disorder (PTLD) is one of the most common life-threatening complications of solid organ transplantation. Its incidence varies depending on the patient's age, the type of immunosuppressant administered, and the type of organ transplanted [11]. Epstein-Barr virus (EBV) infection is present in the majority of PTLD cases and is believed to play a central role in the development of PTLD. Studies have indicated that the pathogenesis of EBV- associated PTLD is related to EBV's ability to transform and immortalize B lymphocytes $[6,8]$. The clinical manifestations of PTLD vary from nonspecific symptoms such as fever, to enlargement of lymph nodes or organ-specific dysfunction. Although the involvement of the central nervous system is reported in $5 \%$ to $30 \%$ of PTLD cases, the occurrence of primary central nervous system PTLD (PCNS-PTLD) is very rare.

A definitive diagnosis of PCNS-PTLD is often challenging to make and requires a thorough physical 
examination, diagnostic imaging, and tissue biopsy. Generally, the prognosis of PCNS-PTLD is poor compared to systemic PTLD, and the treatment recommendations for PCNS-PTLD are not yet well established. For example, the current strategy of dose reduction or discontinuation of immunosuppressive agents is only effective for approximately half of PCNS-PTLD patients, and the efficacy of a singleagent treatment regimen using rituximab is controversial [6]. Therefore, a better understanding of the pathogenesis and clinical features of PCNS PTLD is important for making an accurate diagnosis, which is critical for the proper clinical management of this devastating disease.

We herein report a case of a renal transplant patient who initially presented with a self-limited brain lesion associated with nonspecific neurological alterations that was ultimately found to be PCNS-PTLD.

\section{Clinical summary and pathological findings}

The patient is a 68-year-old woman who underwent kidney transplant due to diabetes-associated end-stage renal disease in 2010. After transplantation, she received an immunosuppressive treatment regime consisting of mycophenolate and tacrolimus for 5 years. She was admitted to our medical center after a fall. Computed tomography (CT) scan of the head detected diffuse hypoattenuation in the subcortical white matter of the left parietal lobe and effacement of the left occipital horn. Magnetic resonance imaging $(\mathrm{MRI})$ of the head revealed a $0.6 \times 0.6 \times 0.5 \mathrm{~cm}$ subcortical mass lesion in the left postcentral gyrus. The lesion showed adjacent edematous change and was suspicious for either a lymphoproliferative disorder or an immunosuppression-associated infection (Fig. 1A). Cerebrospinal fluid (CSF) was collected for infectious disease screening using polymerase chain reaction (PCR) tests, which were positive for EBV at a concentration of $122 \mathrm{IU} / \mathrm{ml}$ ( 1 IU is equal to 0.59 copies) and negative for VZV, HSV, JCV, enterovirus, and toxoplasma. Because of the clinical concern for PTLD, the dose of mycophenolate was reduced and the patient was given a short course of prednisone. The patient underwent a stereotactic brain biopsy, which demonstrated no evidence of a lymphoproliferative disorder (Figs. 2A-B). The lesion showed mild nonspecific reactive changes with mild gliosis, a small number of peri- vascular macrophages, and the presence of very rare CD3-positive lymphocytes. There was no significant increase in B lymphocytes or plasma cells as demonstrated by the negative immunohistochemical stains. The lesion spontaneously resolved (Fig. 1B) and the patient was intermittently followed up by surveillance MRI. Six months later, repeat MRI demonstrated new bilateral parietal enhancing mass lesions with adjacent vasogenic edema (Fig. 1C). Open biopsy of the intracerebral abnormality was performed through image-guided stereotactic craniotomy targeting the area of enhancement in the subcortical location. The biopsy revealed brain parenchyma with a lymphocytic infiltration that consisted of small to medium-sized lymphocytes admixed with numerous large atypical lymphocytes (Fig. 3A). The large atypical lymphocytes were positive for CD20, PAX-5, CD79a, CD10, CD30, BCL-2, BCL-6, and MUM1 (Figs. 3B-E). Moreover, EBV-encoded RNA (EBER) in situ hybridization showed positive staining in the atypical cells (Fig. 3F). The proliferation index (Ki-67 stain) was positive in approximately $50 \%$ of the lymphocytes. These findings were consistent with EBV-positive large B-cell lymphoma. Computed tomography scan with contrast of the chest, abdomen, pelvis, and neck soft tissue showed no evidence of lymphadenopathy or other organ involvement by lymphoma. Bone marrow biopsy was negative for lymphoma involvement. In view of the patient's clinical history of renal transplantation, the diagnosis of PCNS-PTLD, monomorphic type, diffuse large B-cell lymphoma was made. Prompt treatment with the anti-CD20 humanized chimeric monoclonal antibody rituximab (375 mg/m²/week for 4 weeks) was initiated. The patient was ambulatory and maintained good health throughout the chemotherapy. After completing one month of rituximab treatment, follow-up MRI showed resolution of the previously identified biparietal enhancing lesions and an improvement of the adjacent vasogenic edema (Fig. 1D).

\section{Discussion}

Post-transplant lymphoproliferative disorder is a well-recognized complication of solid organ transplantation. The incidence varies depending on many factors, such as the age of the graft recipient, the type and intensity of immunosuppression, and the type of organ transplanted. Post-transplant lymphoproliferative disorder occurs in up to $20 \%$ 

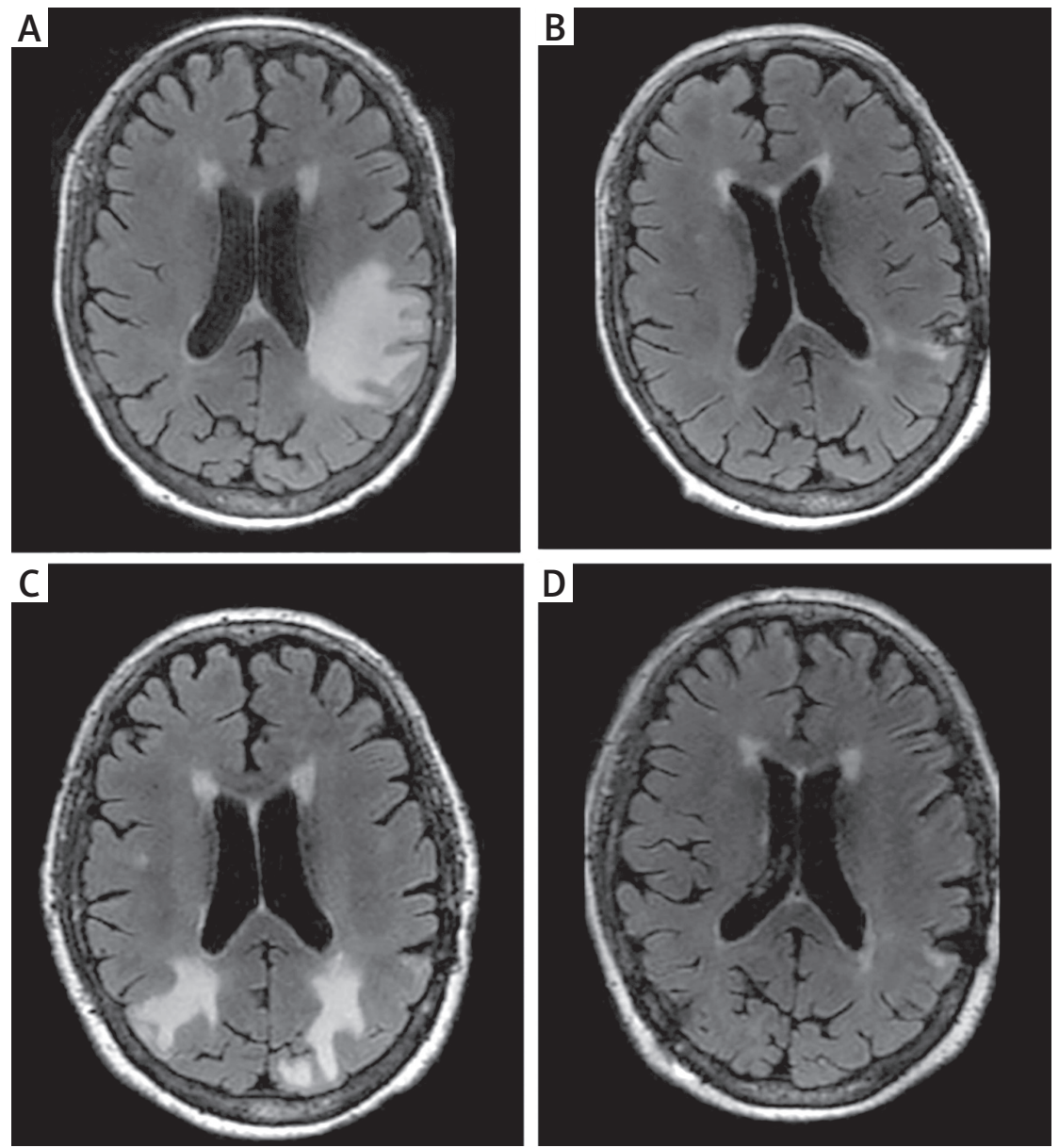

Fig. 1. Magnetic resonance imaging (MRI) examination (Axial T2 FLAIR). A) Magnetic resonance imaging at the time of initial presentation: an enhancing subcortical white matter based lesion in the left postcentral gyrus. There is a mass effect on the left lateral ventricle. B) Spontaneous remission of the lesion (2 months after the initial presentation). There are minimal surrounding $\mathrm{T} 2$ bright signal abnormalities and minimal contrast enhancement. The previously reported mass lesion is not identified. C) Recurrence of the brain lesion (six months after remission of the initial lesion). There are two new enhancing lesions. The first is in the left posterior parietal occipital lobe. The second is located within the right posterior parietal lobe cortex. D) After completing 1 month of rituximab treatment: favorable change with resolution of the previously identified biparietal enhancing lesions and near complete resolution of their adjacent vasogenic edema.
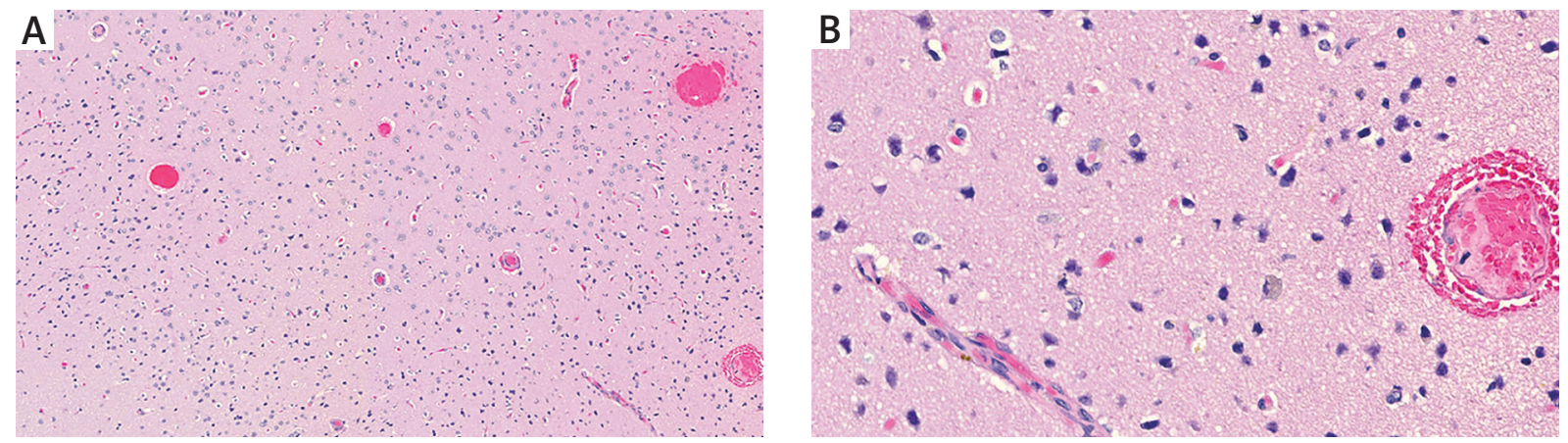

Fig. 2. Photomicrographs of the initial brain biopsy demonstrating a mild nonspecific reactive change with mild gliosis, a small number of perivascular macrophages, and presence of very rare lymphocytes [hematoxylin and eosin (H\&E) stain; original magnification A) $\times 50$; B) $\times 200$ ]. 

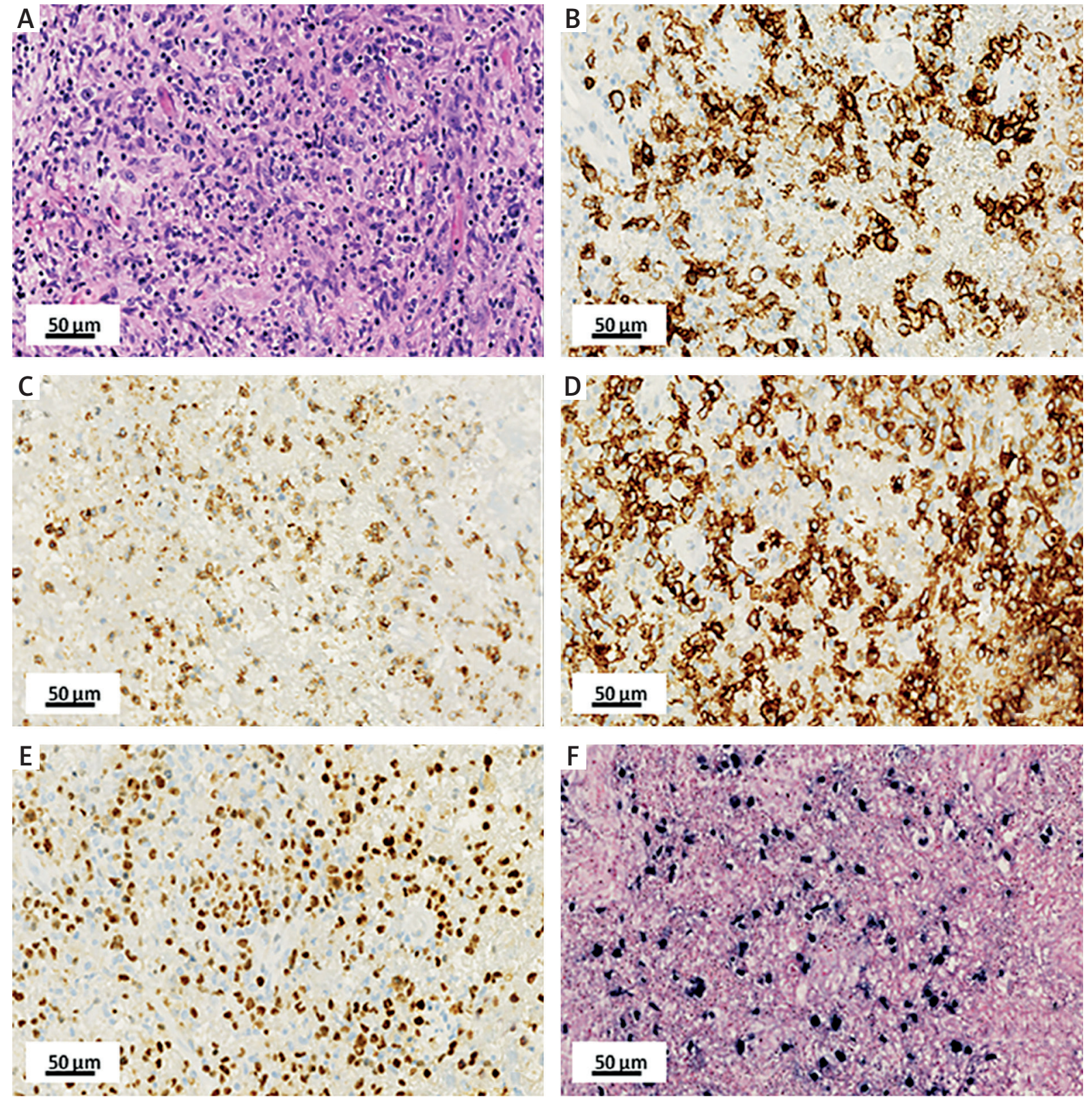

Fig. 3. Photomicrographs of the brain biopsy of the recurrent mass lesions that demonstrate extensive necrosis and marked reactive astrogliosis. There are diffuse infiltrates of small and medium-sized lymphoid cells admixed with atypical large lymphocytes. A) H\&E stain. Immunohistochemical stains show that the infiltrating lymphocytes are positive for CD20 (B), CD10 (C), CD30 (D), and MUM1 (E). In situ hybridization shows positive EBV-encoded RNA in the infiltrating cells $(F)$. Original magnification $(A-F) \times 200$.

of heart, lung and small bowel recipients, but in only $1 \sim 3 \%$ of renal transplant recipients. This difference is most likely related to the less intensive immunosuppressive protocols used in kidney transplantation [10]. The type and duration of immunosuppression have been linked to PTLD risk by affect- ing the host cytotoxic T cells, although it is not clear which immunosuppressive agent has the greatest impact. Mycophenolate mofetil, tacrolimus, cyclosporine, azathioprine, and corticosteroids have been reported to be the major agents contributing to the increased incidence of PTLD [4]. In the current 
case, the patient received a long-term immunosuppressive regimen including mycophenolate and tacrolimus, both of which are associated with a higher risk of developing PTLD [15].

EBV infection is a key contributor to the development of PTLD. In our case, positive EBV was detected by PCR analysis of CSF as well as by in situ hybridization in the lymphoma cells. Primary infection of a previously EBV-seronegative patient during immunosuppression has been identified as the most significant risk factor for the development of PTLD. This may occur through natural exposure or through transmission from an EBV-infected graft [13]. The role of EBV in the pathogenesis of PTLD is likely related to its capacity for incorporation into $B$ lymphocytes during primary infection that may establish lifelong latency. Usually, immunocompetent hosts can mount a humoral response with antibodies and, more importantly, a cellular immune response consisting of cytotoxic T cells [13]. However, the provision of immunosuppressive therapy in organ-transplanted patients alters the innate and adaptive immune responses that prevent $\mathrm{T}$ lymphocytes from controlling the proliferation of EBV-infected $B$ lymphocytes. The resulting high virion peak leads to massive infection of the $B$ cell pool and perhaps other cells not normally infected, such as T cells, NK cells, and memory B cells. The EBV infection ultimately causes secondary events that facilitate lymphocytic immortalization and transformation into PTLD $[6,13]$.

The most commonly used pathologic classification of PTLD is the World Health Organization (WHO) classification, which divides PTLD into four categories: early lesions, polymorphic PTLD, monomorphic PTLD, and Hodgkin lymphoma/Hodgkin lymphoma-like (HL/HL-like) PTLD [8]. Early lesions are characterized by reactive plasmacytic hyperplasia and polyclonal B cell proliferation. Patients can also present with an infectious mononucleosis-type acute illness. Polymorphic PTLD is characterized by the destruction of underlying normal lymphoid architecture by an atypical lymphoid infiltration that usually consists of a heterogeneous cell population with variably sized B cells and plasma cells. Monomorphic PTLD is further subdivided into B-cell and T-cell neoplasms. The majority of monomorphic PTLD cases (> 80\%) are B-cell neoplasms with the most common subtype being diffuse large B-cell lymphoma $[3,8]$. The latter category closely resembles
Hodgkin lymphoma. The histopathological features of the current case are consistent with a monomorphic type PTLD with diffuse large B-cell lymphoma morphology.

The primary goal in treatment of PTLD is to cure the disease, while concomitantly preserving the graft functions. There is no consensus for a treatment model for the optimal management of PTLD due to its clinic-pathologic heterogeneity [7]. In general, the initial therapeutic intervention for PTLD is the reduction in immunosuppression. Rituximab has been used as a single agent or in combination with chemotherapy. Combination chemotherapy is used in patients who fail to respond to reduction in immunosuppression or rituximab and in most patients with advanced disease at presentation [7]. Additional therapeutic options for PTLD include antiviral therapy, cytokine therapy, adoptive immunotherapy, surgery and radiation either as single agent or as combined modality [7].

Primary central nervous system PTLD is a very rare entity defined as an isolated lymphoproliferative disorder of the CNS without evidence of systemic PTLD. In a recent case series that included 910 PTLD cases, $15 \%$ of cases showed CNS involvement, of which less than $50 \%$ were PCNS-PTLD (i.e. only $7 \%$ of all PTLD cases) [2]. The majority of PCNS-PTLD cases were monomorphic B-cell neoplasms, in particular diffuse large B-cell lymphomas that presented with enhancing parenchymal lesions. The time from transplantation to the time of diagnosis of PCNS-PTLD was about 4-5 years, and the median survival was approximately 4 years $[4,6,12]$. Due to its extreme low incidence, treatment of isolated PCNS-PTLD has been challenging and the optimal treatment for PCNS-PTLD has not been established. Although the reduction in immunosuppression has been a mainstay for PTLD, some PCNS-PTLD subjects who had reduction in immunosuppression as the sole treatment did very poorly, suggesting that the reduction in immunosuppression alone may not be sufficient for PCNS-PTLD treatment [11]. Rituximab has shown promising results in systemic PTLD $[1,4,5,14]$. However, the inability of rituximab to cross the blood-brain barrier has raised concerns that the levels of rituximab reached in the central nervous system may not be sufficient to achieve clinical efficacy in PCNS-PTLD treatment. As such, treatment regimens with combination chemotherapy and whole brain radiation have been suggested [4]. However, 
recent studies have indicated that the patients who received rituximab as a single agent demonstrated unexpectedly favorable responses [4,9]. Although experience with the use of this agent is increasing, there is still a need for data from prospective clinical trials. Nevertheless, our patient showed significant clinical improvement with rituximab intervention alone, suggesting that the early, sole use of rituximab is effective and beneficial.

In conclusion, PCNS-PTLD represents a rare and serious complication associated with organ transplantation. The underlying mechanism for the pathogenesis of PCNS-PTLD includes immune system impairment and EBV infection. The early stage of PCNS-PTLD can manifest histopathologically as nonspecific reactive changes, making an early correct diagnosis difficult. This case demonstrates the importance of including PCNS-PTLD as a differential diagnosis in all transplant patients who present with nonspecific CNS symptoms.

\section{Disclosure}

\section{Authors report no conflict of interest.}

\section{References}

1. Bakker NA, van Imhoff GW. Post-transplant lymphoproliferative disorders: from treatment to early detection and prevention? Haematologica 2007; 92: 1447-1450.

2. Buell JF, Gross TG, Hanaway MJ, Trofe J, Roy-Chaudhury P, First MR, Woodle ES. Posttransplant lymphoproliferative disorder: significance of central nervous system involvement. Transplant Proc 2005; 37: 954-955.

3. Castellano-Sanchez AA, Li S, Qian J, Lagoo A, Weir E, Brat DJ. Primary central nervous system posttransplant lymphoproliferative disorders. Am I Clin Pathol 2004; 121: 246-253.

4. Cavaliere R, Petroni G, Lopes MB, Schiff D. Primary central nervous system post-transplantation lymphoproliferative disorder: an International Primary Central Nervous System Lymphoma Collaborative Group Report. Cancer 2010; 116: 863-870.

5. Choquet S, Leblond V, Herbrecht R, Socie G, Stoppa AM, Vandenberghe P, Fischer A, Morschhauser F, Salles G, Feremans W, Vilmer E, Peraldi MN, Lang P, Lebranchu Y, Oksenhendler E, Garnier JL, Lamy T, Jaccard A, Ferrant A, Offner F, Hermine O, Moreau A, Fafi-Kremer S, Morand P, Chatenoud L, Berriot-Varoqueaux N, Bergougnoux L, Milpied N. Efficacy and safety of rituximab in B-cell post-transplantation lymphoproliferative disorders: results of a prospective multicenter phase 2 study. Blood 2006; 107: 3053 3057.

6. Evens AM, Choquet S, Kroll-Desrosiers AR, Jagadeesh D, Smith SM, Morschhauser F, Leblond V, Roy R, Barton B, Gordon LI, Gandhi MK, Dierickx D, Schiff D, Habermann TM, Trappe R. Primary CNS posttransplant lymphoproliferative disease (PTLD): an international report of 84 cases in the modern era. Am J Transplant 2013; 13 1512-1522.
7. Gottschalk S, Rooney CM, Heslop HE. Post-transplant lymphoproliferative disorders. Annu Rev Med 2005; 56: 29-44.

8. Ibrahim HA, Naresh KN. Posttransplant lymphoproliferative disorders. Adv Hematol 2012; 2012: 230173.

9. Jagadeesh D, Woda BA, Draper J, Evens AM. Post transplant lymphoproliferative disorders: risk, classification, and therapeutic recommendations. Curr Treat Options Oncol 2012; 13: 122-136.

10. LaCasce AS. Post-transplant lymphoproliferative disorders. Oncologist 2006; 11: 674-680.

11. Lake W, Chang JE, Kennedy T, Morgan A, Salamat S, Baskaya MK. A case series of primary central nervous system posttransplantation lymphoproliferative disorder: imaging and clinical characteristics. Neurosurgery 2013; 72: 960-970.

12. Paek JH, Kim YR, Jang MH, Hwang EA, Han SY, Park SB. A Brain Tumor from a Posttransplant Lymphoproliferative. J Korean Soc Transplant 2013; 27: 67-71.

13. Thorley-Lawson DA, Gross A. Persistence of the Epstein-Barr virus and the origins of associated lymphomas. N Engl J Med 2004; 350: 1328-1337.

14. Trappe R, Oertel S, Leblond V, Mollee P, Sender M, Reinke P, Neuhaus R, Lehmkuhl H, Horst HA, Salles G, Morschhauser F, Jaccard A, Lamy T, Leithauser M, Zimmermann H, Anagnostopoulos I, Raphael M, Riess H, Choquet S. Sequential treatment with rituximab followed by CHOP chemotherapy in adult B-cell post-transplant lymphoproliferative disorder (PTLD): the prospective international multicentre phase 2 PTLD-1 trial. Lancet Oncol 2012; 13: 196-206.

15. Wimmer CD, Rentsch M, Crispin A, Illner WD, Arbogast H, Graeb C, Jauch KW, Guba M. The janus face of immunosuppression - de novo malignancy after renal transplantation: the experience of the Transplantation Center Munich. Kidney Int 2007; 71: 12711278. 